\title{
A Survey on Ship Intelligent Cabin
}

\author{
Shuli JIA ${ }^{\mathrm{a}}$, Fuxin $\mathrm{WANG}^{\mathrm{a}, 1}$, Mengchao $\mathrm{DONG}^{\mathrm{b}}$ and Liyong $\mathrm{MA}^{\mathrm{b}}$ \\ a Automation Engineering Department, Shanghai Marine Diesel Engine Research \\ Institute, Shanghai, China \\ ${ }^{\mathrm{b}}$ School of Information Science and Engineering, Harbin Institute of Technology, \\ Weihai, China
}

\begin{abstract}
At present, intelligent ship has become a new hot spot of international maritime research and development. In order to achieve the purpose of safety, reliability, energy conservation, environmental protection, economy and efficiency, Intelligent ship integrates modern information technology, artificial intelligence technology and other new technologies with traditional ship technology. In this essay, the rapid development of intelligent cabin technology in recent years is surveyed. The intelligent cabin technology and the development trend of the current technology is analyzed, and the possible development direction in the future is pointed out. Collection, transmission and storage of sensor data are summarized. The development of status perception of cabin equipment and environment is discussed. Data-driven intelligent applications are summarized, and the development in the future is discussed. Intelligent cabin system is a data driven information system, which involves data acquisition, communication, storage, analysis, visualization and other rich content. Using the data provided by intelligent ship, more and more intelligent applications will be developed for ships.
\end{abstract}

Keywords. Intelligent ship, ship cabin, survey, data-driven application

\section{Introduction}

At present, intelligent ship has become a new hot spot of international maritime research and development. In order to achieve the purpose of safety, reliability, energy conservation, environmental protection, economy and efficiency, Intelligent ship integrates modern information technology, artificial intelligence technology and other new technologies with traditional ship technology [1-3]. According to the Intelligent Ship Specification of China Classification Society, the intelligent cabin is one of the important modules in the six intelligent modules, which provides vital support for the normal operation of the intelligent ship.

Intelligent technology can be divided into three levels, computational intelligence, perceptual intelligence and cognitive intelligence. The intelligent cabin mainly includes the cabin's perceptual intelligence and cognitive intelligence. Perceptual intelligent of ship cabin technology is a series of process. Sensors are used to obtain equipment and environmental information of ship cabin, and then the effective feature information is processed and understood. Cognitive intelligent of ship cabin technology is to further enhance the perceptual information to a cognizable level, such as memory, understanding,

\footnotetext{
${ }^{1}$ Fuxin WANG, Corresponding author, Automation Engineering Department, Shanghai Marine Diesel Engine Research Institute, Shanghai, China; E-mail: 18801909355@163.com.
} 
planning, decision-making and so on. Data analysis or intelligent diagnosis methods are used in the state assessment and fault diagnosis of ship cabin systems and equipment, which is an important part of ship intelligence.

Marine ship cabin equipment mainly includes three categories as shown in Fig.1, they are power and control equipment, anti-pollution equipment and other auxiliary equipment. Power and control equipment includes main engine, auxiliary engine, boiler and steering gear. Anti-pollution equipment includes oil-water separators, incinerators, and domestic sewage treatment devices. Other auxiliary equipment includes oil separator, water generator, pumping device, etc. It can be seen that ship cabin equipment is the core of ship power and operation, so the use of intelligent technology to realize the perception and cognition of these core equipment of ship cabin will play an irreplaceable role in the development of ship technology.

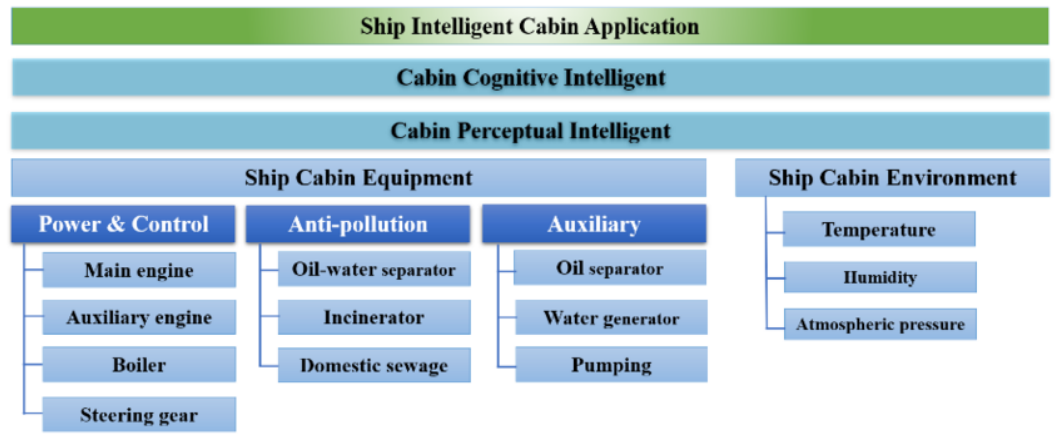

Figure 1. Architecture of intelligent ship cabin.

Although there have been a lot of literature on the research of ship intelligent cabin, but there is not a survey on these researches of intelligent cabin. The main contribution of this paper is as follows. First, the rapid development of intelligent cabin technology in recent five years is surveyed. As far as we know, there is no existing comprehensive study on intelligent cabin technology. Second, the development trend of the current technology on intelligent cabin is analyzed, and the possible development direction in the future is pointed.

\section{Collection, Transmission and Storage of Sensor Data}

The intelligent cabin mainly uses sensor, data acquisition module and communication equipment to collect data from equipment and system of ship cabin. The specific acquisition equipment and systems include main engine system, gearbox system, pitch control propeller system, fuel system, lubricating oil system, cooling water system, exhaust system, air system, steering gear system, oil separator system, boiler system and auxiliary engine system, as well as video monitoring information [4-5].

For data transmission, communication methods include serial port, USB and ethernet. Fieldbus technology is also used frequently, especially in control networks containing sensors and actuators. CAN bus is commonly used as field bus in ship systems [5].

The data storage development of intelligent ships has gone through two stages. The first stage is centralized storage, generally using relational databases [4-5]. The second 
stage mainly adopts distributed storage technology [2,6]. The real-time monitoring of a small number of equipment can be realized through the central database. But when managing a large number of devices, distributed storage is more flexible and efficient. Distributed database engine is the core of the system, which is responsible for SQL parsing, rewriting and execution, and managing many storage nodes at the bottom. In the process of cabin data management, different scale database clusters can be flexibly constructed. By dividing the business data into different database storage nodes, the database has less pressure when facing massive data. By distributing the user's SQL request to the sub-workstations of each node for execution, the computing resources of each node are fully utilized, and the operating efficiency of the server cluster is improved as well.

\section{Status Perception of Cabin Equipment and Environment}

In summary, for marine ship cabin equipment such as diesel generator sets and propulsion motors, the collected signals can be classified into fast varying signals and slowly varying signals.

For slowly varying signals, such as speed, temperature, pressure and other thermal signals, the sampling frequency of these signals is low. Such data can be obtained through communication with control equipment or directly sampled, and the collected data are directly stored in the database. The feature extraction of slowly varying signal is mainly based on time domain analysis. The operation status of equipment is sensed through peak-peak, root mean square, kurtosis and other methods [7-8].

For fast varying signals, generally, the feature extraction is mainly based on frequency domain analysis and time-frequency domain analysis, and the operating status of the equipment is sensed through changes in the frequency spectrum and related parameters. Vibration signal is the most familiar signal [9], it can be analyzed through time domain, frequency domain, and time-frequency domain feature analysis. And the fusion analysis of feature information can be used to perceive the operating status of the device.

Multi-sensor information fusion plays an important role in device state perception. The complementary or redundant information of multiple sensors in time or space, and comprehensive judgment based on the information of multiple sensors, can effectively improve the accuracy of status perception [10-12]. Engine room equipment, especially the main power equipment, is a highly coupled system of thermodynamics, dynamics, and control. This makes the composite faults of gas circuits and mechanical components show the characteristics of coupling of fault characteristics and complex fault mechanisms, and it is difficult to characterize equipment faults with a single knowledge. In order to improve the fault perception accuracy, it is necessary to study the feature fusion method on the basis of the coupling characteristics of the equipment operation mechanism in the multi-source information fusion stage [10-12]. Using a full range of multiple attributes to redundantly describe faults may be a future research direction.

Fault diagnosis is one of the most studied problems in device state awareness. The problem of fault diagnosis is also representative in perception problems [12-16]. The following is a summary of the research methods and development of state perception, represented by the research of fault diagnosis. Fault diagnosis methods mainly include analytical method, signal processing method and knowledge-based method. The method 
based on analytical method can determine whether the model changes in the actual operation process and the degree of change, so it could determine whether the fault occurs and the possible causes of the fault [8]. The signal processing method directly uses the signal model to process and analyze the original signal, extract the corresponding fault characteristic information and judge whether it is faulty [9]. The knowledge-based method does not require a quantitative mathematical model, it will comprehensively use the information of the diagnosis target and use expert knowledge to complete the diagnosis. A lot of research has focused on this method, and the methods used include neural networks [9,13], fuzzy mathematics [12], and other intelligent method including machine learning $[14,15]$ are used here.

\section{Data-driven Intelligent Applications}

Due to the large number of ship information systems and the huge amount of information transmission between each other, the intelligent cabin can provide data-driven support for applications. These applications include big data $[1,2,14]$ and artificial intelligence methods [16-19]. There are important research advances in the intelligent collision avoidance decision-making application [18-21].

The failure assessment and prediction of ship cabin equipment has been widely studied [22-29]. Generally speaking, fault prediction is divided into state prediction and life prediction. Classical fault prediction methods mainly include function fitting, Kalman filter, particle filter, grey model, neural network, et al. Recently, data-driven intelligent methods have been widely employed for fault diagnosis.

It can be seen that scholars have carried out in-depth research on fault diagnosis and prediction of marine engine [22], and the application of new intelligent algorithm is also constantly innovating. At present, the related research work mainly focuses on the accuracy of fault classification, and there are few research results on the optimization of algorithm cost and calculation efficiency. However, in the actual operation of the ship, the response speed and calculation cost are the key indicators to evaluate the fault diagnosis and prediction system. Therefore, how to accurately diagnose and predict the fault based on low cost and high response speed is a problem that cannot be ignored in the real ship application.

Energy efficacy is another hot topic for intelligent applications of ship [28-32]. Researches on intelligent integrated navigation and obstacle avoidance are also emerging. It is foreseeable that applications in this area will explode rapidly after the wide citation of smart cabin. We expect these intelligent applications could take root and grow.

\section{Conclusions}

This paper summarizes the development of intelligent cabin technology. Intelligent cabin is the integration of modern information technology, artificial intelligence technology with traditional ship technology. Intelligent cabin system is a data driven information system, which involves data acquisition, communication, storage, analysis, visualization and other rich content. By providing data support, many intelligent applications based on data become possible. And the data and applications can provide strong support for user operation, maintenance and decision-making. With the development of data sensing 
technology, 5G communication technology, real-time data storage technology, visualization technology, artificial intelligence and other advanced technologies, intelligent cabin system will play an increasingly important role in the actual ship.

\section{References}

[1] Cao M, Guo C. Key technologies of big data and its development in intelligent ship. In Proceedings of International Conference on Robotics and Artificial Intelligence (ICRAI); 2017 Dec 29- 31; Shanghai, China: ACM Press; p. 61-65.

[2] Jia S, Ma L, Zhang S. Big data prototype practice for unmanned surface vehicle. In: Proceedings of the 4th International Conference on Communication and Information Processing; 2018 Nov 2-4; Qingdao, China: ACM Press; p. 43-47.

[3] Im I, Shin D, and Jeong J. Components for smart autonomous ship architecture based on intelligent information technology. In Proceedings of International Conference on Mobile Systems and Pervasive Computing; 2018 Aug 13-15; Calle Gomera, Spain: Elsevier Press; p. 91-98.

[4] Li G, Deng X, Zhou M, et al. Research on data monitoring system for intelligent ship. Lecture Notes in Electrical Engineering. 2020 Nov; 634(1):234-241.

[5] Luo X. Research on communication technology of ship integrated monitoring system based on OPC. In Proceedings of International Conference on Intelligent Transportation, Big Data and Smart City; 2020, Jan 11-12; Vientiane, Laos: IEEE Press; p. 528-531.

[6] Barsocchi P, Ferro E, Rosa D, et al. E-cabin: A software architecture for passenger comfort and cruise ship management. Sensors. 2019 Nov; 19(22): 49781-19789.

[7] Chen L, Wang S, Wang H, et al. Model construction and application of machinery fault diagnosis of ships based on technology of resonant demodulation. Acta Technica CSAV. 2017 Feb; 62(2):477-485.

[8] Kowalski J, Krawczyk B, Woniak M. Fault diagnosis of marine 4-stroke diesel engines using a one-vsone extreme learning ensemble. Engineering Applications of Artificial Intelligence. 2017 Jan; 57(1):134141.

[9] Li Z, Chen J, Zi Y, He S. A sensor-dependent vibration data driven fault identification method via autonomous variational mode decomposition for transmission system of shipborne antenna. Sensors and Actuators, A: Physical. 2018 Jan; 279(1):376-389.

[10] Zhang C, Guo C, Guo M. Information fusion based on artificial intelligence method for SINS/GPS integrated navigation of marine vessel. Journal of Electrical Engineering and Technology. 2020 May;15(3):1345-1356.

[11] Xu H, Zhou Z, Han X, Li W. Self-tuning weighted fusion estimation method for intelligent ship. Journal of Huazhong University of Science and Technology (Natural Science Edition). 2019 Mar; 47(3):25-30.

[12] Zhu Y, Geng L. Research on SDG fault diagnosis of ocean shipping boiler system based on fuzzy granular computing under data fusion. Polish Maritime Research. 2018 Jan; 25(1):92-97.

[13] $\mathrm{Wu}, \mathrm{G}$. Fault detection method for ship equipment based on BP neural network. In Proceedings of International Conference on Robots and Intelligent System; 2018 May 26-27; Changsha, China: IEEE Press; p.556-559.

[14] Yang M, Shi W. Research on fault diagnosis of ship power system based on improved particle swarm optimization neural network algorithm. In Proceedings of IEEE Advanced Information Technology, Electronic and Automation Control Conference; 2018 Oct 12-14; Chongqing, China: IEEE Press; p.108113.

[15] Wang Z, Wang Y. Yang X. Research on multi-agent fault diagnosis system for ship integrated power system. In Proceedings of Chinese Automation Congress; 2018 Nov 30- Dec 2; Xi'an, China: IEEE Press; p.3137-3140.

[16] Feng D, Li Y. Research on intelligent diagnosis method for large-scale ship engine fault in nondeterministic environment. Polish Maritime Research, 2017 May; 24(S3):200-206.

[17] Ma L, Xie W, Huang H. Convolutional neural network based obstacle detection for unmanned surface vehicle. Mathematical Biosciences and Engineering. 2020 Jan; 17(1):845-861.

[18] Arendt R, Kopczyski A, Spychalski P. Centralized and distributed structures of intelligent systems for aided design of ship automation. In Proceedings of International Conference on Information Systems Architecture and Technology; 2017 Sep 17-19; Szklarska Poland: Springer Press; p.310-319.

[19] Losiewicz Z, Nikoczuk P, Pielka D. Application of artificial intelligence in the process of supporting the ship owner's decision in the management of ship machinery crew in the aspect of shipping safety. In Proceedings of International Conference on Knowledge-Based and Intelligent Information Engineering Systems; 2019 Sep 4-6; Budapest Hungary: Elsevier Press; p.2197-2205. 
[20] Sedova N, Sedov V, Bazhenov R. Intelligent system of automatic ship course operation based on the neural network governor mechanism. In Proceedings of International Conference on Industrial Engineering, Applications and Manufacturing; 2019 Mar 25-29; Sochi Russia: IEEE Press; p.1-5.

[21] Wang C, Zhang X, Chong L, et al. Research on intelligent collision avoidance decision-making of unmanned ship in unknown environments. Evolving Systems. 2019 Apr; 10(4):649-658.

[22] Du L, Banda O, Kujala P. An intelligent method for real-time ship collision risk assessment and visualization. In Proceedings of International Conference on Collision and Grounding of Ships and Offshore Structures; 2019 Oct 21-23; Lisbon Portugal: CRC Press; p.293-300.

[23] Borkowski P. Inference engine in an intelligent ship course-keeping system. Computational Intelligence and Neuroscience. 2017 Nov; 2017(11): 1-8.

[24] Gao M, Shi G, Li S. Online prediction of ship behavior with automatic identification system sensor data using bidirectional long short-term memory recurrent neural network. Sensors. 2018 Dec; 18(12):1-12.

[25] Huang B, Zou Z. Online prediction of ship roll motion in irregular waves using a fixed grid wavelet network. Journal of Ship Mechanics. 2020 Jun; 24(6):693-705.

[26] Jeon M, Noh Y, Shin Y, et al. Prediction of ship fuel consumption by using an artificial neural network. Journal of Mechanical Science and Technology. 2018 Dec; 32(12):5785-5796.

[27] Nie Z, Yuan Y, Xu D, et al. Research on support vector regression model based on different kernels for short-term prediction of ship motion. In Proceedings of International Symposium on Computational Intelligence and Design; 2019 Dec 14-15; Hangzhou, China: IEEE Press; p.61-64.

[28] Ye Y, Cheng Y, Lai J, et al. Fault prediction model of ship unloader based on improved association rules. China Mechanical Engineering. 2019 Oct; 30(20):2463-2472.

[29] Orihara H. Tsujimoto M, Performance prediction of full-scale ship and analysis by means of on-board monitoring. Part2: Validation of full-scale performance predictions in actual seas. Journal of Marine Science and Technology (Japan). 2018 Apr; 23(4):782-801.

[30] Prera L, Mo B. Machine intelligence based data handling framework for ship energy efficiency. IEEE Transactions on Vehicular Technology. 2017 Oct; 66(10):8658-8666.

[31] Kalikatzarakis M, Geertsma R, Boonen E, et al., Ship energy management for hybrid propulsion and power supply with shore charging. Control Engineering Practice. 2018 Jan; 76(1):133-154.

[32] Tan Z, Chen S, Lin H, et al. Ship intelligent energy efficiency management system design. In Proceedings of International Ocean and Polar Engineering Conference; 2019 Jun 16-21; Honolulu, United states: International Society of Offshore and Polar Engineers; p.4412-4417. 